\title{
Fibonacci Spiral Arranged Ultrasound Phased Array for Mid-Air Haptics
}

\author{
$1^{\text {st }}$ Adam Price \\ Ultrahaptics Ltd. \\ Bristol, UK \\ adam.price@ultrahaptics.com
}

\author{
$2^{\text {nd }}$ Benjamin Long \\ Ultrahaptics Ltd. \\ Bristol, UK \\ ben@ultrahaptics.com
}

\begin{abstract}
Ultrasound phased arrays are most commonly used in non-destructive testing and medical diagnostic applications. More recently mid-air haptic technology has utilized the acoustic radiation force to produce skin deformations and thus recreate the sensation of touch. The acoustic radiation force is the timeaveraged force exerted by an acoustic wave on a medium and can be spatially and temporally controlled using electronically steered phased ultrasonic arrays. An important problem with most phased arrays has been the presence of grating lobes arising from the periodicity of array element spacing and arrangement that are detrimental to haptic sensations. We show that by arranging the elements of an array in a Fibonacci spiral, one can suppress grating lobes. We discuss the benefits of fabricating Fibonacci spiral arrays as opposed to sparse arrays. We also discuss the benefits of removing grating lobes for mid-air haptic applications such as control panels for automotive dashboards and immersive experiences in VR.
\end{abstract} tics

Index Terms-phased array, Fibonacci spiral, ultrasound, hap-

\section{INTRODUCTION}

Many engineering designs take inspiration from nature, from a kingfisher's beak on the front of a Japanese bullet train to a burdock burrs' microscopic hooks on a velcro strap. Biomimicry is a powerful tool and the arrangement of transducers in a phased array can take inspiration from the arrangement of seeds on a sunflower. The most efficient packing of seeds of the same size would be a honeycomb pattern. However as a sunflower is continuously growing new seeds from the centre it needs a pattern that can evolve while still making the most economical use of space [1]. The arrangement that achieves this is the Fibonacci spiral. Imagine placing seeds one by one around a central axis at angular intervals of any exact fraction of $\pi$ and at an increasing radial distance. Eventually the pattern repeats and will be shaped like a star with a number of straight arms equal to the denominator. Placing seeds separated by the golden angle, as it is irrational and does not have a denominator by definition, both packs seeds efficiently and does not exhibit these straight arms, so no lines of symmetry appear in a Fibonacci spiral

This project has received funding from the European Unions Horizon 2020 research and innovation programme under grant agreement No 737087, LEVITATE Project pattern. For elements in an ultrasonic array the aforementioned straight arms result in grating lobes, making the Fibonacci spiral pattern an effective method of removing grating lobes.

Another method to remove grating lobes is element spacing; if the element spacing in an array is less than $\lambda / 2$ grating lobes will not be produced. Various approaches have been attempted to acheive this desired spacing. For example Konetzke et al. [2] used acoustic wave guides although this has the disadvantage of adding thickness and mounting complexity to an array. Korobov et al. placed transducers into Fresnel zone rings [3] with each excited out of phase, but with this design there was no control of the focal point position. Kazys et al. [4] constructed an array where the piezoelectric crystals vibrated lengthwise along the thinnest dimension to achieve less than $\lambda / 2$ spacing, however the design is not very efficient at coupling to the air. Sparse arrays are also another important array design that through random positioning of elements avoids introducing lines of symmetry [5], however as elements are spread out more in this design the peak amplitude of a focal point is dramatically diminished.

\section{Linear Phased ArRays}

The phenomenon of grating lobes can be explained and explored through the Huygens model, a ray tracing model originally used in the field of optics. In the Huygens model each point on a wavefront is regarded as a source of waves expanding from that point, these wavelets are assumed to not go through any physical changes and to interact with each other linearly. Starting with the simplest case of a linear phased array of point sources one can characterize the beam directivity of an array with $N$ elements, a given spacing $d$, and a steering angle $\theta_{S}$. Equation (1) defines the far field pressure at angles from the centre of the array, normalised at the steering angle:

$$
H(\theta)=\left|\frac{\sin \left[\frac{\pi d\left(\sin \theta_{s}-\sin \theta\right)}{\lambda} N\right]}{N \sin \left[\frac{\pi d\left(\sin \theta_{s}-\sin \theta\right)}{\lambda}\right]}\right|
$$

Although there are differences between a steering beam (converging waves to an angle) and a focusing beam (converging waves to a point), equation (1) can be used to illustrate 


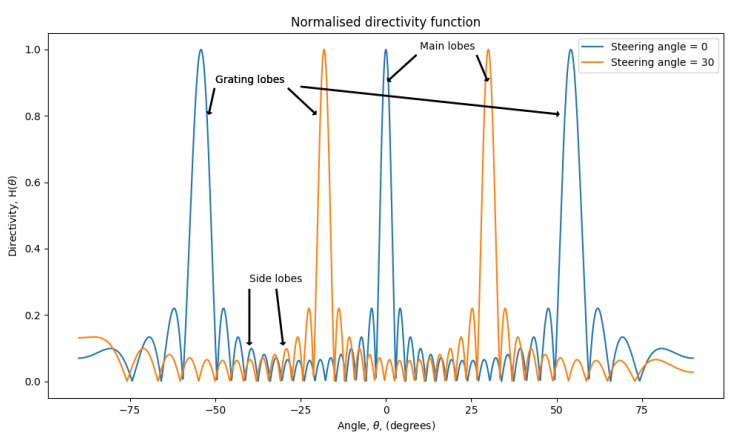

Fig. 1. Normalised directivity function for an evenly spaced linear array steered at angles of $0^{\circ}$ and $30^{\circ}$

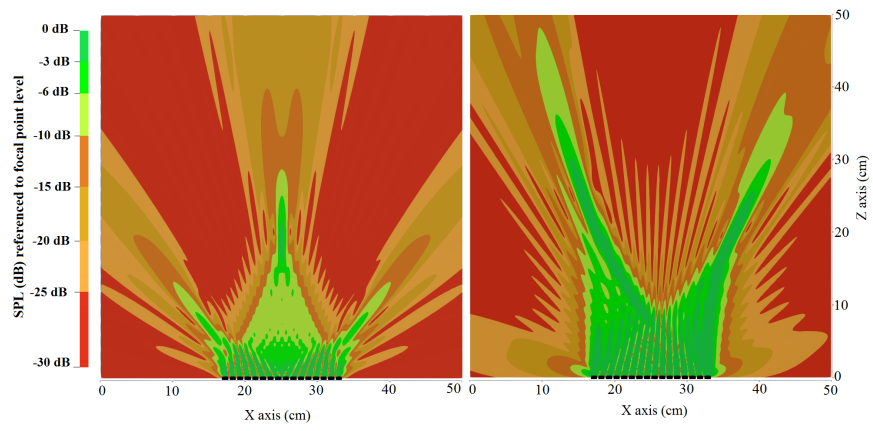

Fig. 2. Acoustic field slice for focal points $20 \mathrm{~cm}$ above the array at $0^{\circ}$ and $30^{\circ}$ from the centre

important features of array characteristics. Figure 1 shows an example of two different steering angles $\left(0^{\circ}\right.$ and $\left.30^{\circ}\right)$, with a linear array of 16 transducers $(N=16)$ with an ultrasonic carrier wavelength of $8.575 \mathrm{~mm}$ and element spacing of $10.5 \mathrm{~mm}$. The main lobes appear along the steering angle, while side lobes cover almost all of the other angles. The grating lobes have exactly the same magnitude as the main lobe. In practice the amplitude directivity of the transducers will cause grating lobes to have lower amplitudes than the main lobe.

Figure 2 shows the same array configuration (linear) as Figure 1 along with the same two steering angle examples, but now the array is focussing. One can see from Figure 2 how the non-uniform directivity of a transducer affects the amplitude of the grating lobes. The non-uniform directivity means grating lobes are considerably lower in amplitude when the focal point is positioned in the middle of the array compared to when the focal point is angled off to one side.

A technique described in Long et al. [6] shows how one can control the amplitudes of all the transducers in the array to suppress grating lobes by using a minimum-norm solution. Its effects are similar to apodization wherein the closer the transducer is to the desired focus the higher the amplitude.

\section{Fibonacci Spiral Phased Arrays}

A Fibonacci spiral phased array can be created by placing the $n^{\text {th }}$ transducer at the polar coordinate given by $r(n)=\sqrt{n}$ and $\theta(n)=2 \pi \phi n$, where $\phi=(1+\sqrt{5}) / 2 \approx 1.618$. Figure
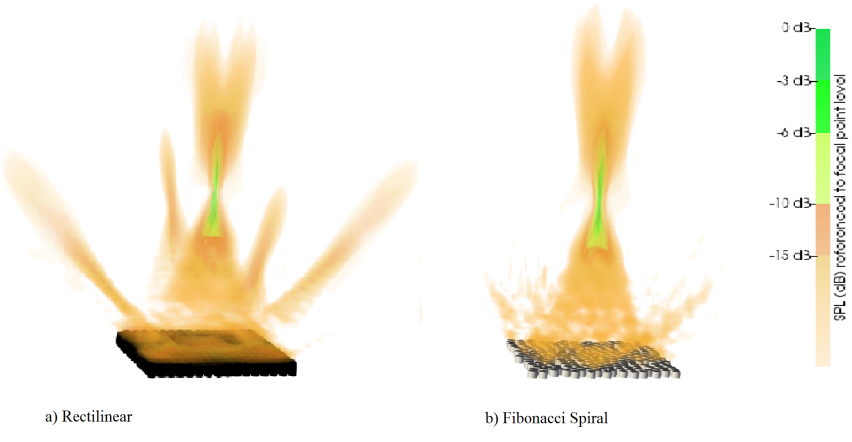

Fig. 3. Simulations of acoustic fields for a rectilinear and fibonacci spiral array

3 shows simulations of the resulting focused acoustic field from 2D rectilinear and Fibonacci spiral arrays. The absence of grating lobes is clear for the Fibonacci spiral, in contrast to the rectilinear array. Note that the apodization technique can also be seen clearly in the rectilinear array as the grating lobes are now more than $10 \mathrm{~dB}$ below the focal point level. Due to electronic connector limitations and the transducers' physical size, a small perturbation is applied to the exact Fibonacci spiral design to obtain a physical layout of 191 transducers mounted on a $16 \times 16 \mathrm{~cm}$ PCB (see Figure 5).

Having found a design that removes grating lobes, it is important to check the comparative performance of the fabricated array. An optimal packing is always a regular lattice, which for transducers larger in diameter than $\lambda / 2$ would always produce grating lobes. So the best packing of elements that does not is by definition less densely populated. This results in a lower output power per unit area; in this case a reduction in output power of approximately $\sim 25 \%$ (as the sunflower design uses 191 transducers in place of 256). The reduction in density is not completely disadvantageous, as measures to heatsink the transducers can be reduced for this case. A further metric for assessing the performance of focussing arrays is the Array Performance Indicator (API) defined in equation (2).

$$
\mathrm{API}=\frac{\text { Volume }_{-6 \mathrm{~dB}}}{\lambda^{3}}
$$

Holmes et al. [7] originally used the API to determine an array's ability to detect and image a point-like reflector. Here, we are using it to determine the array's ability to focus to the smallest possible point in space. This point can then be detected by a human hand as the smallest building block of a mid-air haptic sensation. Note that Holmes et al. were only concerned with the area the array could focus on, whereas in mid-air haptic applications depth is also important so the API metric has been extended to account for the focus volume. The smaller the API value, the better the array is at focusing to a point.

\section{EXPERIMENTAL INVESTIGATIONS}

A scanning laser Doppler vibrometer (SLDV) was used to investigate grating lobe occurrence while the microphone 


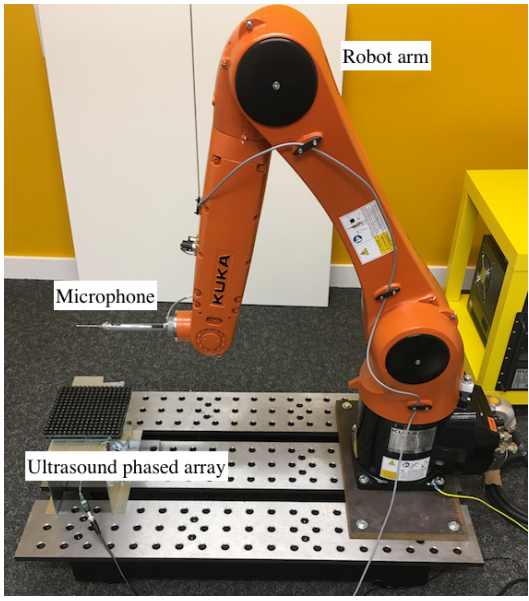

Fig. 4. Microphone and robot arm experimental set up
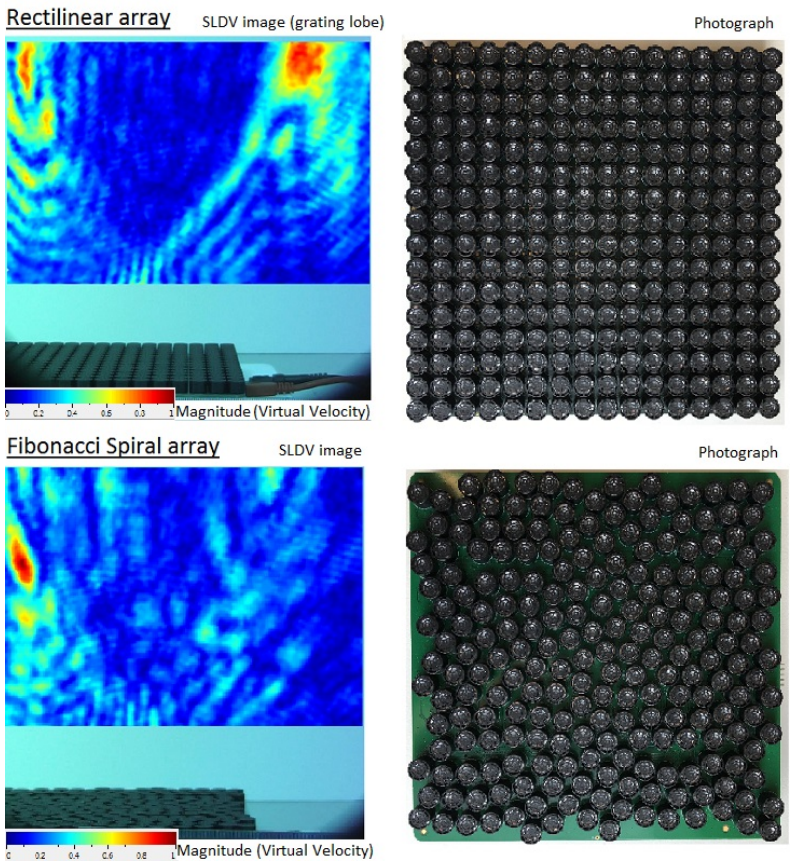

Fig. 5. Acoustic field images and photographs of Fibonacci Spiral and Rectilinear arrays

was used to investigate the focal point profiles to determine the API of each array. Although quantified sound field data may be gathered using an SLDV system, for a focussing beam the experimental setup required becomes impractical [8]. Therefore for this experiment the images obtained are 2D projections of the 3D sound field. As the laser traverses the acoustic field, pressure variations change the local refractive index of the air which are then detected by the SLDV as the vibration of a "virtual" surface, when the beam reflects back into the device. The root-mean-squared (RMS) velocity of the vibration has a known relationship to acoustic pressure [9]. For the microphone measurements, a pressure field (Brüel \& Kjær Type 4138-L-015) microphone with a Brüel \& Kjær Type 2829
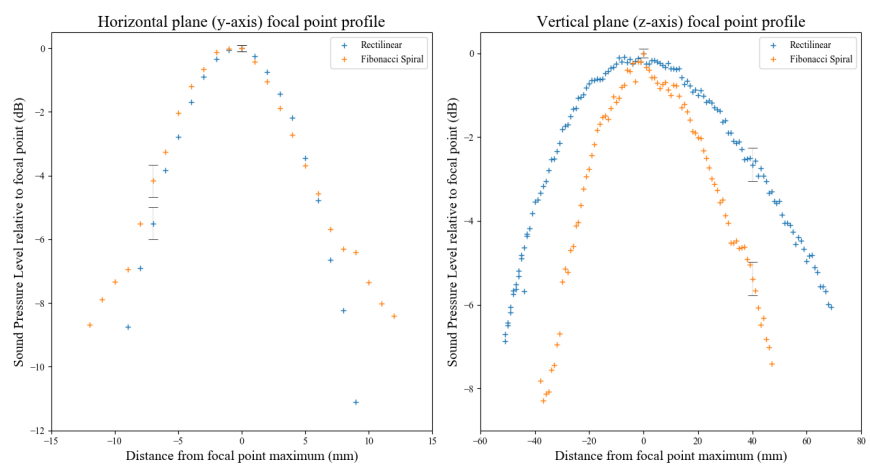

Fig. 6. Experimental results of focal point profiles for rectilinear and Fibonacci Spiral arrays

power supply and the PXIe-4310 National Instruments Analog Input Module were used to acquire the data. With sound pressure level being very sensitive to position a robot arm (KUKA KR10 1100 sixx) was used to position the microphone in various locations in the acoustic field (Figure 4).

\section{A. Scanning Laser Doppler Vibrometry Results}

Figure 5 shows the acoustic field images for the two types of array; focussing to a point $5 \mathrm{~cm}$ off centre, $20 \mathrm{~cm}$ above each array. A grating lobe to the top right of the image is clearly visible in the rectilinear array acoustic field image. SLDV images are created by using a phase reference (in this case an internal electrical $40 \mathrm{kHz}$ clock signal produced by the logic board of the array was used) that stitches all the measurement points together. Figure 5 shows the RMS velocity, but the data capture can also be set with a timer and with an appropriate trigger more locations of focal points can be investigated. By using the phase sequence that moves a focal point in a path across the array, a measurement may be triggered so the changes in grating lobes with different focal point positions can be observed.

\section{B. Microphone Results}

Microphone measurements were taken in the acoustic fields of the Fibonacci Spiral array and the Rectilinear array $(16 \times 16)$. All scans involved having the focal point centred $20 \mathrm{~cm}$ above the array. The focal point was located by selecting the point of maximum pressure in the field around the predicted position of the focus. After refining its location, the microphone was then scanned in millimetre increments through the focal point to determine its shape. Random fluctuations in the field produced errors in the measurements, temperature changes in the room are one likely cause of this error. Error bars show \pm 1 standard deviation calculated from the distribution of these fluctuations when measured at the focal point, a point $6 \mathrm{~mm}$ outside the focus in the horizontal plane and $40 \mathrm{~mm}$ above the focus in the vertical plane in Figure 6. All plots are shown to a relative decibel scale for ease of comparison. The results in Figure 6 were then used to calculate an API for each array, shown in Table I. 
TABLE I

API RESULTS

\begin{tabular}{|c|c|}
\hline Array type & API \\
\hline Rectilinear $(16 \times 16)$ & 17.9 \\
\hline Fibonacci Spiral & 17.2 \\
\hline
\end{tabular}

\section{DiscusSiON}

The SLDV images provide strong evidence that the Fibonacci spiral array successfully eliminates grating lobes. This is a practical solution for mid-air haptic applications where the presence of grating lobes can confuse the user with unwanted haptic sensations. Although sparse arrays can also eliminate such artefacts, in a real-world implementation where space is at a premium, such an array would be impractical. Further, we have shown that the extent of the focus of a Fibonacci spiral array is more compact in the $z$-axis, leading to an advantage in localisation of the ultrasonic mid-air haptics. The $x$ and $y$ axes are unchanged as these depend more on the geometric footprint [10], and not the transducer arrangement. This said, perceptual tests, similar to Ito et al. [11], would have to be run to determine whether the changes in profile of the focal point can be detected by the user of a mid-air haptic device.

As mid-air haptics becomes mainstream, it is more and more necessary to understand the design space of ultrasonic arrays and how ramifications of those design choices impact the user experience. Applications for the technology include VR and automotive user interfaces, where its relevance has recently been demonstrated with an experimental validation of a significant reduction in eyes-off-the-road glance times compared with touchscreen center console interfaces [12]. As the objective of the introduction of mid-air haptic interfaces in this situation is to reduce distraction and increase safety, in such haptic interfaces, it would be unacceptable for grating lobes to distract the user from their task or the road.

In a virtual reality setting, the single differentiator between success and failure is immersion. A misplaced element, stray effect or grating aberration can break the illusion. Again a grating lobe from a rectilinear array can easily interfere with the experience, generating phantom haptic effects that the content designer did not intend.

\section{CONCLUSION}

Through simulation and experiment in this paper, the Fibonacci spiral array has been shown to eliminate grating lobes while keeping the design compact. When measured the focal point shape was comparable between the Fibonacci spiral array and the rectilinear array in the horizontal plane, with the Fibonacci spiral array yielding a smaller focus in the vertical plane (with respect to the surface of the array).

Its performance was checked further using the API metric that showed no appreciable differences in total focal point size and thus array quality between the two designs.

For automotive applications size is critical and a compact design that can be placed either on the dashboard or centre console is more desirable than a sparse array, the absence of grating lobes is also very important when designing haptic sensations to indicate different functions for the on-board car control panel. A grating lobe can be confused for a focal point, with the wrong selection made in the menu user interface. For VR applications a sensation caused by a grating lobe in an unintended location can have a detrimental effect on the immersion of the user.

For future work, we would like to assess more completely changes in the API indicator through the space of all possible focus positions, to understand the variance with different device layouts and how this affects design choices. In conclusion, from a practical standpoint the Fibonacci spiral array yields improvements in mid-air haptic systems that bring the technology as a whole closer to every day life.

\section{ACKNOWLEDGMENT}

This project has received funding from the European Unions Horizon 2020 research and innovation programme under grant agreement No 737087.

\section{REFERENCES}

[1] R. Dixon, New Scientist, 17 December 1981.

[2] E. Konetzke et al. "Phased array transducer for emitting $40-\mathrm{kHz}$ aircoupled ultrasound without grating lobes, in Ultrasonics Symposium (IUS), 2015 IEEE International, pp. 14, IEEE, 2015.

[3] A. I. Korobov, M. Y. Izosimova, and S. A. Toschov, "Development of ultrasound focusing discrete array for air-coupled ultrasound generation, Physics Procedia, vol. 3, no. 1, pp. 201207, 2010.

[4] R. J. Kazys, R. Sliteris, and J. Sestoke, "Air-coupled low frequency ultrasonic transducers and arrays with PMN32\% PT piezoelectric crystals, Sensors, vol. 17, no. 1, p. 95, 2017.

[5] S. G. Goss et al. "Sparse random ultrasound phased array for focal surgery." IEEE Transactions on Ultrasonics Ferroelectrics and Frequency Control 43.6: 1111-1121, 1996.

[6] B. Long, et al. "Rendering volumetric haptic shapes in mid-air using ultrasound." ACM Transactions on Graphics (TOG) 33.6: 181, 2014.

[7] C. Holmes, B. W. Drinkwater, and P. D. Wilcox, "Post-processing of the full matrix of ultrasonic transmitreceive array data for non-destructive evaluation, NDT E Int., vol. 38, no. 8, pp. 701711, Dec. 2005.

[8] A. Torras-Rosell, S. Barrera-Figueroa, F. Jacobsen, Sound field reconstruction using acousto-optic tomography, The Journal of the Acoustical Society of America 131, 3786-3793, 2012.

[9] R. Malkin, T. Todd and D. Robert, "A simple method for quantitative imaging of 2D acoustic fields using refracto-vibrometry." Journal of Sound and Vibration, 2014.

[10] G. B. Airy, "On the diffraction of an object-glass with circular aperture." Transactions of the Cambridge Philosophical Society 5: 283, 1835.

[11] M. Ito, et al. "High spatial resolution midair tactile display using 70 kHz ultrasound." International Conference on Human Haptic Sensing and Touch Enabled Computer Applications. Springer, Cham, 2016.

[12] K. Harrington et al. "Exploring the Use of Mid-Air Ultrasonic Feedback to Enhance Automotive User Interfaces." Proceedings of the 10th International Conference on Automotive User Interfaces and Interactive Vehicular Applications. ACM, 2018. 